\title{
Predição de dados de sensoriamento visando eficiência energética de redes de sensores sem fio
}

\author{
Charles M. P. Almeida ${ }^{1}$, Geymerson S. Ramos ${ }^{1}$, Andre L. L. Aquino ${ }^{1}$ \\ ${ }^{1}$ Instituto da Computação - Universidade Federal de Alagoas (UFAL) \\ Maceió - AL - Brasil \\ \{charles.pedrosa, geymerson.r, alla.lins\}@gmail.com
}

\begin{abstract}
This article aims to compare prediction models applied to wireless sensor networks in order to increase energy efficiency in data gathering. ARIMA, SVM and ANN models were deployed in an application to collect temperature data and for each model, an evaluation of it's energy savings contribuition has been made. The measurements were performed by real devices and it was possible to observe the performance of the models for a set of collected data in an office environment. In this study case, ARIMA model showed a better performance when compared to SVM and ANN in terms of energy efficiency.
\end{abstract}

Resumo. Este artigo tem como objetivo comparar modelos de predição de dados de sensoriamento em redes de sensores sem fio com a finalidade de economizar energia na coleta de dados. Modelos ARIMA, SVM e ANN foram utilizados em uma aplicação de coleta de dados de temperatura e avaliados quanto à economia de energia proporcionada. As medições foram realizados por dispositivos reais e foi possivel observar o desempenho dos modelos para um conjunto de dados de sensoriamento em um ambiente de escritório. Para este estudo de caso, o modelo ARIMA apresentou melhor desempenho em relação ao SVM e ANN em termos de eficiência energética.

\section{Introdução}

As redes de sensores sem fio (RSSFs) [Akyildiz et al. 2002] permitem o monitoramento e controle de variáveis físicas como temperatura, som, luz, vibração, pressão, movimento ou poluição] [Ruela et al. 2009]. Nas RSSFs os nós sensores possuem geralmente energia limitada e a comunição sem fio destes sensores pode ser considerada a principal forma de consumo. Portanto, reduzir a sobrecarga de comunicação pode ser o método mais eficaz para economizar energia nestes sistemas [Liu et al. 2005].

Em [Thamizhselvi and Mary 2016] é apresentada uma classificação das técnicas de eficiência energéticas nas RSSFs. Redução dos Dados é uma destas técnicas e seu foco está na redução do montante de dados produzidos, processados e transmitidos. Neste trabalho a redução do consumo de energia se dá através da redução da quantidade de transmissões entre nó sensor e na estação-base. A redução é obtida através da supressão do envio de parte dos dados coletados no nó sensor e sua substituição por dados gerados em um modelo de predição na estação-base. Diversos trabalhos nesta linha de pesquisa foram realizados [Liu et al. 2005, Wang et al. 2008, Moghadam and Keshmirpour 2011]. Em [Liu et al. 2005], utiliza-se um modelo ARIMA para predizer os valores dos dados 
coletados. A acurácia e eficiência da predição para esta utilização pode ser melhor ou pior a depender do modelo que é utilizado.

Este artigo tem como objetivo comparar a eficiência energética em RSSFs a partir da supressão da comunição entre nós utilizando modelos de predição de dados. Os modelos utilizados na comparação foram: i) ARIMA (do inglês, Autoregressive Integrated Moving Average) é um dos mais populares modelos lineares em previsão de séries temporais [Zhang 2003]; ii) SVM (do inglês, Support Vector Machines) [Cortes and Vapnik 1995] é um algoritmo de mineração de dados e considerado um dos métodos mais robustos e precisos entre os conhecidos algoritmos de mineração; iii) e ANN (do inglês, Artificial Neural Network) reune seus conhecimentos detectando padrões e relacionamentos nos dados e aprendendo (ou sendo treinados) através da experiência, não da programação [Zhang et al. 1998].

Este artigo se organiza da seguinte forma: Seção 2 apresenta trabalhos relacionados com a pesquisa de eficiência energética e predição de dados utilizando os modelos ARIMA, SVM e ANN. Seção 3 trata dos modelos utilizados. Seção 4 detalha a aplicação desenvolvida para este estudo de caso. Seção 5 discute os resultados da comparação entre os modelos. Seção 6, conclusões e futuras direções de trabalho.

\section{Trabalhos Relacionados}

Diversos trabalhos apresentam abordagens para predição de leituras de sensores com o objetivo de economizar energia. Especificamente em [Liu et al. 2005] o objetivo é economizar energia na coleta de informações em redes de sensores sem fio. A metodologia empregada se baseia em manter os nós sensores da transmissão de informações redundantes, através da predição pelo nó coletor (estação-base). O ARIMA é utilizado como modelo para predição, propondo um esquema de coleta de informações eficiente em termos energéticos. A eficácia do método proposto apresentado no trabalho é demonstrada com uma aplicação real baseada na plataforma MICA2.

O trabalho de [Wang et al. 2008] investiga o problema da eficiência energética e propõe um método de organização eficiente de energia com previsão de séries temporais. A organização das redes de sensores sem fio está formulada para rastreamento de alvo. Modelo de alvo, modelo de multi-sensor e modelo de energia são definidos. A posição alvo futura é derivada de modelos de média móvel autorregressiva (ARMA). Os resultados experimentais verificam que a combinação do modelo ARMA e a decomposição do modo empírico podem estimar a posição alvo de forma eficiente e a economia de energia é alcançada pelo método de organização proposto.

A abordagem de [Moghadam and Keshmirpour 2011] se baseia no argumento que uma das maneiras de reduzir o consumo de energia dos nós sensores é reduzir o número transmissões sem fio entre nós por predição dupla.

Em linhas gerais existem uma diversidade de técnicas e soluções para o problema aqui apresentado. Contudo, diferente do que apresentamos nesse trabalho, não existe um estudo comparativo aprofundado do impacto das técnicas para a predição de dados de sensoriamento visando a eficiência energética em RSSFs. 


\section{Modelos de Predição}

\subsection{Modelo ARIMA}

A metodologia de Box-Jenkins para a previsão se baseia no ajuste de modelos denominados ARIMA para séries temporais de valores observados. A diferença entre os valores gerados pelos modelos e os valores observados resulta em séries de resíduos de comportamento aleatório em torno de zero. A análise de séries temporais usa os dados históricos para desenvolver um modelo para a previsão de valores. Neste trabalho os dados históricos são os valores coletados pelos nós sensores e enviados para a estação-base, onde é executado um modelo ARIMA para a predição dos dados futuros.

O modelo ARIMA [Liu et al. 2005] é amplamente utilizado para séries temporais univariadas. O mesmo é composto de três termos: O termo Auto Regressivo (AR), que é uma regressão linear do valor atual da série contra um ou mais valores anteriores. Ele captura a dependência do valor atual e seus valores anteriores mais próximos; O termo de Média Móvel (MA), que é introduzido para capturar a influência de choques aleatórios no futuro, uma vez que geralmente uma série temporal pode receber choques aleatórios em um ambiente ruidoso e pode memorizar os choques recebidos anteriormente por um tempo [Liu et al. 2005]; e o terceiro termo Integrador (I) para remover o efeito da não estacionariedade com a diferenciação dos dados, formando assim um modelo ARIMA. Vale destacar que o modelo ARMA, também utilizado, é a combinação dos dois primeiros termos, uma vez que assume que a série é estacionária. No entanto, nem sempre isso acontece, logo o terceiro termo precisa ser introduzido.

O modelo $\operatorname{ARMA}(p, q)$ refere-se ao modelo com $p$ termos auto-regressivos e $q$ termos na média móvel. O termo $\operatorname{AR}(p)$, de ordem $p$, pode ser descrito como

$$
y_{t}=c+a_{1} y_{t-1}+\ldots+a_{p} y_{t-p}+u_{t}
$$

onde $a_{1}, \ldots, a_{p}$ são parâmetros, $c$ é uma constante, e a variável aleatória $u_{t}$ é ruído branco. O termo $\mathrm{MA}(q)$, de ordem $q$, pode ser descrito como

$$
y_{t}=\mu+u_{t}+m_{1} u_{t-1}+\ldots+m_{q} u_{t-q}
$$

onde $m_{1}, \ldots, m_{q}$ são parâmetros do modelo, $\mu$ é a expectativa para $y_{t}$ (normalmente 0 ), $u_{t}, u_{t-1}, \ldots, u_{t-q}$ são termos de erro de ruído branco. $\mathrm{O}$ modelo contendo os $\operatorname{AR}(p)$ e $\mathrm{MA}(q)$ pode ser descrito como

$$
y_{t}=c+a_{1} y_{t-1}+\ldots+a_{p} y_{t-p}+u_{t}+m_{1} u_{t-1}+\ldots+m_{q} u_{t-q}
$$

Quando $\operatorname{AR}(p), \operatorname{MA}(q)$ e $\operatorname{ARMA}(p, q)$ são aplicados em alguns casos onde os dados mostram evidência de não-estacionariedade, uma diferenciação inicial deve ser aplicada para reduzir esta não-estacionariedade, chegando-se assim a um modelo ARIMA. Modelos não sazonais são geralmente denotados por $\operatorname{ARIMA}(p, d, q)$, onde $p$ é a ordem do modelo $\mathrm{AR}, d$ é o grau de diferenciação e $q$ é a ordem do modelo MA [Yuan et al. 2016].

\subsection{Modelo SVM}

As máquinas de vetor de suporte (SVMs) implementam o princípio da minimização de risco estruturada (SRM, do inglês, Structured Risk Minimization) em vez do princípio de 
minimização de risco empírico implementado pela maioria dos tradicionais modelos de rede neural. O conceito mais importante de SRM é a minimização de um limite superior para o erro de generalização em vez de minimizar o erro de treinamento. Com base nesse princípio, as SVMs conseguem uma ótima estrutura de redes [Pai and Hong 2005].

Os modelos SVMs geram a função de regressão aplicando um conjunto de funções lineares de altas dimensões. A função de regressão SVM é formulada da seguinte forma [Pai and Lin 2005]

$$
y=w \phi(x)+b
$$

onde $\phi(x)$ é chamado de recurso (feature em inglês), que é oriunda do mapeamento não linear do espaço de entrada de $x$. Os coeficientes $w$ e $b$ são estimados pela minimização

$$
R(C)=C \frac{1}{N} \sum_{i=1}^{N} L_{\varepsilon}\left(d_{i}, y_{i}\right)+\frac{1}{2}\|w\|^{2}
$$

onde

$$
L_{\varepsilon}(d, y)=\left\{\begin{array}{lr}
|d-y|-\varepsilon & |d-y| \geq \varepsilon \\
0 & \text { outros }
\end{array}\right.
$$

$C$ e $\varepsilon$ são parâmetros prescritos. O primeiro termo $L_{\varepsilon}(d, y)$ é chamado de função de perda $\varepsilon$-itensiva. $\mathrm{O} d_{i}$ é o valor atual da série no período de índice $i$. Esta função indica que o erro abaixo $\varepsilon$ não é penalizado. O termo $C \frac{1}{N} \sum_{i=1}^{N} L_{\varepsilon}\left(d_{i}, y_{i}\right)$ é o erro empírico. O segundo termo, $\frac{1}{2}\|w\|^{2}$ mede a planicidade da função. $C$ avalia o trade-off entre o risco empírico e a planicidade do modelo. Introduzindo as variáveis de folga positivas $\zeta$ e $\zeta^{*}$, que representam as distâncias dos valores reais para os valores limites de $\varepsilon$-tubo. A equação 5 é transformada na seguinte forma

Minimizar:

$$
R\left(w, \zeta, \zeta^{*}\right)=\frac{1}{2} w w^{T}+C^{*}\left(\sum_{i=1}^{N}\left(\zeta_{i}+\zeta_{i}^{*}\right)\right)
$$

Sujeito a:

$$
\begin{aligned}
w \phi\left(x_{i}\right)+b_{i}-d_{i} & \leq \varepsilon+\zeta_{i}^{*} \\
d_{i}-w \phi\left(x_{i}\right)-b_{i}-d_{i} & \leq \varepsilon+\zeta_{i} \\
\zeta_{i}, \zeta_{i}^{*} & \geq 0
\end{aligned}
$$

com $i=1,2, \ldots, N$. Introduzindo o multiplicador de Lagrange e maximixando a função dupla da Equação 7, temos

$$
\begin{aligned}
R\left(\alpha_{i}-\alpha_{i}^{*}\right)= & \sum_{i=1}^{N} d_{i}\left(\alpha_{i}-\alpha_{i}^{*}\right)-\varepsilon \sum_{i=1}^{N}\left(\alpha_{i}-\alpha_{i}^{*}\right)- \\
& \frac{1}{2} \sum_{i=1}^{N} \sum_{j=1}^{N}\left(\alpha_{i}-\alpha_{i}^{*}\right) \times\left(\alpha_{j}-\alpha_{j}^{*}\right) K\left(x_{i}, x_{j}\right)
\end{aligned}
$$


com as restrições

$$
\sum_{i=1}^{N}\left(\alpha_{i}-\alpha_{i}^{*}\right)=0
$$

sendo $0 \leq \alpha_{i} \leq C$ e $0 \leq \alpha_{i}^{*} \leq C$ com $i=1,2, \ldots, N$. Na Equação 11, $\alpha_{i}$ e $\alpha_{i}^{*}$ são chamados de multiplicadores de Lagrange [Pai and Lin 2005]. Eles satisfazem as igualdades

$$
\begin{aligned}
\alpha_{i} * \alpha_{i}^{*} & =0 \\
f\left(x, \alpha, \alpha^{*}\right) & =\sum_{i=1}^{l}\left(\alpha_{i}-\alpha_{i}^{*}\right) K\left(x_{i}, x_{j}\right)+b
\end{aligned}
$$

onde $K\left(x, x_{i}\right)$ é a função Kernel. O valor da Kernel é igual ao produto interno dos vetores $x_{i}$ e $x_{j}$ no espaço do recurso $\phi\left(x_{i}\right)$ e $\phi\left(x_{j}\right)$, de modo que $K\left(x_{i}, x_{j}\right)=\phi\left(x_{i}\right) * \phi\left(x_{j}\right)$. Qualquer função que satisfaça a condição de Mercer pode ser usada como função Kernel [Pai and Lin 2005]. A condição Mercer é a regra para determinar uma Kernel admissível de vetor de suporte [Xiao-Ying et al. 2009]. Neste estudo utilizamos função Kernel do tipo radial.

\subsection{Modelo ANN}

O tipo de rede neural utilizada neste trabalho foi a multi-layer perceptrons (MLP). As redes MLP são usadas na predição devido a sua capacidade de mapeamento arbitrário de entrada e saída. Uma rede MLP é tipicamente composta por várias camadas. A primeira camada serve como entrada e a última é uma camada de saída onde a solução do problema é obtida. A camada de entrada e a camada de saída são separadas por uma ou mais camadas intermediárias, chamadas camadas ocultas. Os elementos das camadas adjacentes (neurônios artificiais ou nós) são totalmente conectados por arcos acíclicos de uma camada inferior a uma camada superior. A Figura 1 dá um exemplo de um MLP totalmente conectado com uma camada oculta [Zhang et al. 1998]. Para o problema de predição, as

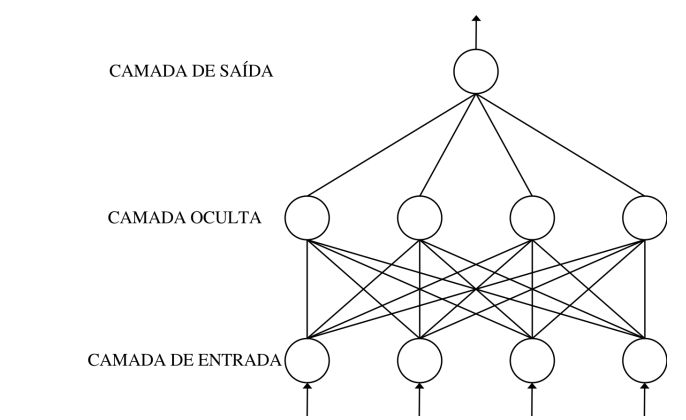

Figura 1. Uma típica rede neural feedfoward

entradas são geralmente as variáveis independentes ou preditoras. A relação funcional estimada pela ANN pode ser escrita como

$$
y=f\left(x_{1}, x_{2}, \ldots, x_{p}\right)
$$


onde $x_{1}, x_{2}, \ldots, x_{p}$ são as $p$ variáveis independentes e $y$ é a variável dependente. Nesse sentido, a rede neural é funcionalmente equivalente a um modelo de regressão não linear. Para um problema de predição de séries temporais, as entradas são tipicamente as observações passadas e a saída é o valor futuro. A ANN executa o seguinte mapeamento de funções

$$
y_{t+1}=f\left(y_{t}, y_{t-1}, \ldots, y_{t-p}\right)
$$

onde $y_{t}$ é a observação no tempo $t$. Assim, o moledo ANN é equivalente ao modelo autorregressivo não linear para problemas de predição de séries temporais.

O modelo ANN necessita ser treinado antes de realizar a tarefa desejada. O treinamento é o processo de determinar os parâmetros do modelo (geralmente ajuste dos pesos) e ele pode ocorrer de forma supervisionada ou não supervisionada. Para o problema de predição em séries temporais, um padrão de treinamento consiste em um número fixo de observações defasadas da série. Ou seja, suponha que temos $N$ observações $y_{1}, y_{2}, \ldots, y_{N}$ no conjunto de treinamento e precisamos da predição em um passo à frente. Usando um modelo ANN com $n$ nós de entrada, temos $N-n$ padrões de treinamento. O primeiro padrão de treinamento será composto de $y_{1}, y_{2}, \ldots, y_{n}$ como entradas e $y_{n+1}$ como a saída alvo. O segundo padrão de treinamento conterá $y_{2}, y_{3}, \ldots, y_{n+1}$ como entradas e $y_{n+2}$ como a saída desejada. Finalmente, o último padrão de treinamento será $y_{N-n}, y_{N-n+1}, \ldots, y_{N-1}$ para entradas e $y_{N}$ para o alvo. Comumente uma função objetivo utilizada durante o processo de treinamento é dada por

$$
E=\frac{1}{2} \sum_{i=n+1}^{N}\left(y_{i}-\alpha_{i}\right)^{2}
$$

onde $\alpha_{i}$ é a saída real da rede e o valor $1 / 2$ é o fator de simplificação da expressão de derivadas computadas no algoritmo de treinamento.

\section{Aplicação}

Para coleta dos dados de temperatura, 5 dispositivos da plataforma MICAz ${ }^{1}$ foram utilizados, sendo 4 destes utilizados como nós (sensores) e 1 como estação-base. A estação-base foi conectada a um computador de mesa que atuará como gateway. No gateway é feito processamento de dados e nele são executados os modelos ARIMA, SVM e ANN. Os nós foram colocados próximos um do outro para mensurar simultaneamente sob as mesmas condições do ambiente e o distanciamento de cada nó para a estação-base é de aproximadamente 5 centímetros. Um diagrama da aplicação é apresentado na Figura 2, onde é observada a relação entre nó, estação-base e o gateway.

Cada dispositivo foi programado conforme seu papel na RSSF, sendo que, os nós sensores mensuram e transmitem dados de temperatura diretamente para a estação-base, que por sua vez os recebe e os transmite para o gateway. Ao receber os dados, o gateway processa e gera um conjunto de predições para um dos modelos citados, isto é, um conjunto de predições para o ARIMA, SVM ou ANN, a depender do nó que realizou a transmissão.

\footnotetext{
${ }^{1}$ http://www.memsic.com/userfiles/files/Datasheets/WSN/micaz_datasheet-t.pdf, acessado em março de 2018
} 


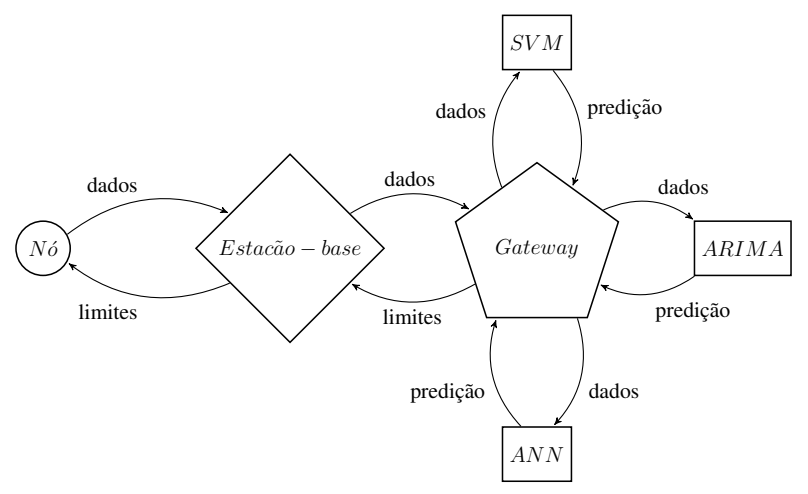

Figura 2. Diagrama da aplicação

Cada conjunto de predições possui 30 valores gerados a partir das 30 últimas temperaturas transmitidas e, com os valores preditos, calcula-se uma média. Com a média, são definidos limites superiores e inferiores que serão utilizados como parâmetros para determinar quando um nó deve ou não transmitir um pacote de dados. O limite superior é dado pela soma de um valor $\delta$ à média de um conjunto de predições e, de maneira análoga, o limite inferior é dado pela subtração deste valor da média. O gateway é responsável por calcular estes limites e transmiti-los para para um determinado nó da rede através da estação-base.

Cada nó mensura e armazena localmente dados de temperatura de segundo em segundo e, ao final de 30 segundos, os transmite para a estação-base, caso os limites calculados pelo gateway tenham sido ultrapassados. Para fins de comparação, as transmissões foram feitas de forma contínua (o nó sempre transmitindo a cada 30 segundos) e intermitente (transmite às vezes). Dos quatro nós sensores utilizados, além da estaçãobase, um destes realizou transmissão continuamente e os outros três transmitiram apenas no caso de ultrapassagem dos limites superiores ou inferiores, sendo que, um nó teve seus limites definidos com base nas predições do ARIMA, um nas predições do SVM e o terceiro baseado em predições do ANN. Caso um nó ultrapasse seu limite superior ou inferior, um pacote de medições é transmitido, e o gateway calcula novos limites e os transmite para o nó.

O gateway mantém versões virtuais dos nós físicos da rede, ambos funcionam em sincronia de modo que, sempre que um nó físico não realizar uma transmissão, o gateway assumirá que os valores das medições estão dentro dos limites calculados e o nó virtual armazenará um conjunto de temperaturas preditas por seu respectivo algoritmo. Este conjunto de temperaturas será armazenado repetidamente até que um novo pacote de dados seja recebido e novas predições sejam realizadas. Nesta situação, os valores reais de temperatura recebidos serão armazenados e, passados 30 segundos, se um novo pacote não for recebido, o nó virtual armazenará periodicamente valores de predição baseados nos valores reais. A curva final de temperatura de um nó é portanto constituída de valores reais e preditos.

\section{Avaliações}

O erro dos modelos de predição propostos foi avaliado pelo MAPE (do inglês, Mean Absolute Percentage Error), MAD (do inglês, Mean Absolute Deviation) e pelo NRMSE 
(do inglês, Normalized Root Mean Square Error), como determinado pelas Equações 18, 19 e 20, respectivamente. Foram comparadas a curva real de temperatura e as curvas preditas nos nós sensores. O resultado destas avaliações pode ser verificado na tabela 1.

$$
\begin{aligned}
M A P E & =\frac{1}{n} \sum_{i=1}^{n}\left|\frac{d_{i}-y_{i}}{d_{i}}\right| \times 100 \% \\
M A D & =\frac{\sum_{i=1}^{n}\left|d_{i}-y_{i}\right|}{n} \\
N R M S E & =\sqrt{\frac{\sum_{i=1}^{n}\left(d_{i}-y_{i}\right)^{2}}{\sum_{i=1}^{n} d_{i}^{2}}}
\end{aligned}
$$

A amostra avaliada corresponde a um período de aproximadamente 6 horas com 23.070 medições de temperatura em cada um dos nós. Apesar de estarem na mesma localidade, os nós apresentaram variações nas temperaturas mensuradas. O nó com predições dadas pelo modelo ARIMA realizou o menor número de transmissões, seguido pelo SVM e por último, ANN.

\begin{tabular}{l|c|c|c}
\hline & ARIMA & SVM & ANN \\
\hline MAPE & $1,98 \%$ & $2,07 \%$ & $2,75 \%$ \\
MAD & 0,47 & 0,49 & 0,65 \\
NRMSE & 0,02 & 0,02 & 0,03 \\
\hline
\end{tabular}

Tabela 1. Avaliação de Erro dos Modelos

Foi realizada também avaliação com a finalidade de comparar o desempenho dos modelos ARIMA, SVM e ANN na predição de dados de sensoriamento visando eficiência energética na RSSF. Durante o fornecimento de potência elétrica de um conjunto de baterias para uma aplicação, o descarregamento destas baterias pode ser graficamente visualizada através de uma curva onde temos no eixo $x$ a variável tempo e no eixo $y$ a variável tensão elétrica. Durante o ciclo de funcionamento, a tensão elétrica decai de forma suave até valores toleráveis pela aplicação, e então, diminui abruptamente fazendo com que o sistema não opere mais, devido ao fato de não estar com o nível de tensão inadequado. Esta curva pode ser chamada de curva de descarregamento das baterias. Na Figura 3, lado esquerdo, temos uma amostra de aproximadamente $6 \mathrm{~h}$ onde podemos ver parte do decaimento da tensão das baterias em cada um dos quatro nós durante o sensoriamento da temperatura. Ainda na Figura 3, lado direito, são apresentadas as curvas de Potência Entregue em cada um dos nós. A curva de Potência Entregue é proporcional ao quociente do quadrado da tensão pela resistência do circuito e interna das baterias.

Para a avaliação do modelo que apresenta maior eficiência energética no sensoriamento de temperatura foi verificada a quantidade de energia, correspondente às diferenças das áreas sob as curvas do gráfico da Figura 3, de cada um dos modelos em relação ao nó que sempre envia seus dados, desta forma, verificando quanto a supressão do envio de dados trouxe de economia de energia para as baterias.

O modelo ARIMA apresentou, para esta amostra, uma maior eficiência energética na RSSF. Na Tabela 2 podemos verificar os valores percentuais de economia de energia 

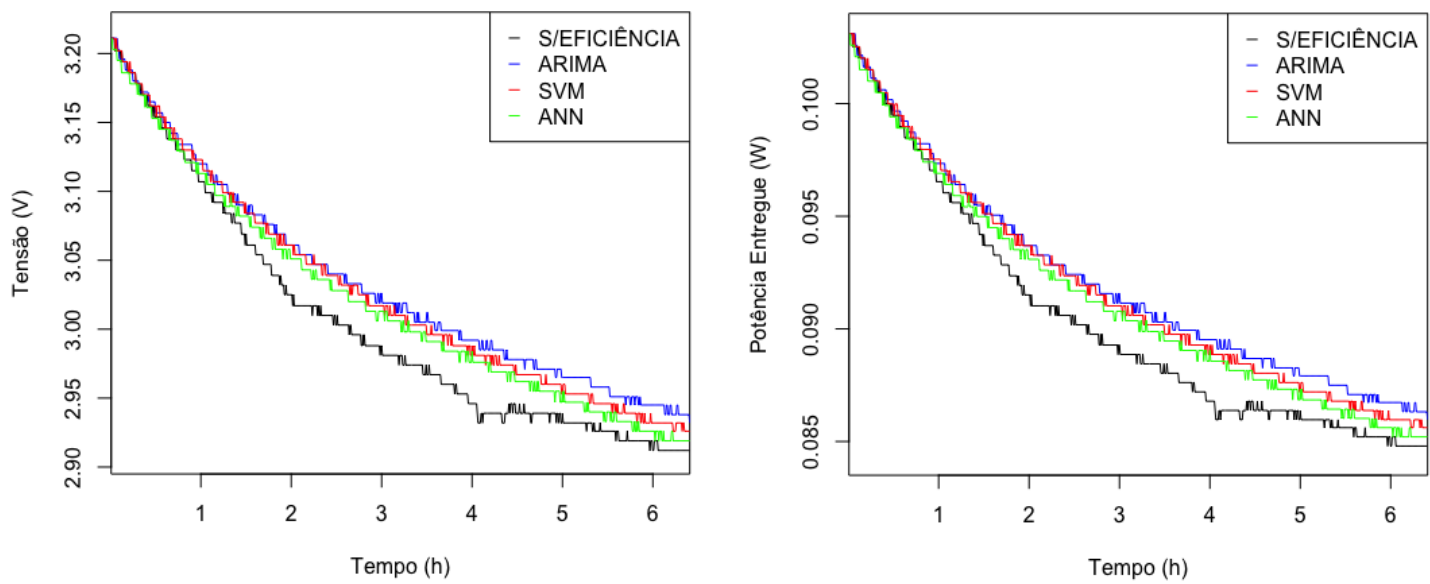

Figura 3. Curva de Descarga das Baterias e Curva de Potência Entregue

\begin{tabular}{l|c|c|c}
\hline & ARIMA & SVM & ANN \\
\hline Redução do Consumo de Energia & $1,90 \%$ & $1,53 \%$ & $1,07 \%$ \\
\hline
\end{tabular}

Tabela 2. Eficiência Energética dos Modelos

para as baterias no intervalo de tempo supracitado. O nó com o modelo ARIMA economizou cerca de $2 \%$ de energia quando comparado ao nó que sempre transmite. Vale salientar que nesta amostra de aproximadamente 6 horas, o valor da constante $\delta$ incrementada ou somada para os limites superiores e inferiores respectivamente era de apenas 0,03 graus Celcius e, a depender destes limites e do intervalo de tempo da coleta, o valor de economia certamente será bem maior.

\section{Conclusão e trabalhos futuros}

Nas RSSFs o gasto de energia elétrica devido à transmissão de dados entre os nós sensores e a estação-base, em geral, a maior parcela do consumo. Diversas abordagens no sentido de suprimir o envio das informações existem e uma delas é a supressão do envio de dados através da predição dos valores num modelo matemático [Liu et al. 2005]. Neste artigo comparamos três modelos ARIMA, SVM e ANN para verificar qual apresenta maior eficiência energética.

O modelo ARIMA apresentou, para as amostras coletadas, um desempenho superior aos modelos SVM e ANN, sendo mais preciso nas predições e economizando mais energia das baterias da RSSF. Trabalhos anteriores já avaliaram o modelo ARIMA isoladamente aplicado para eficiência energética de RSSF, no entanto este trabalho ratifica a sua qualidade quando comparado com outros modelos.

Os modelos se mostraram sensíveis quanto à escolha dos seus parâmetros e uma abordagem de otimização ou utilizando modelos híbridos pode contribuir para melhorar a análise em estudos futuros.

\section{Agradecimentos}

Agradecemos ao CNPq, FAPEAL e FAPESP pelo apoio financeiro. 


\section{Referências}

Akyildiz, I., Su, W., Sankarasubramaniam, Y., and Cayirci, E. (2002). Wireless sensor networks: a survey. Computer Networks, 38(4):393 - 422.

Cortes, C. and Vapnik, V. (1995). Support-Vector Networks. Machine Learning, 20(3):273-297.

Liu, C., Wu, K., and Tsao, M. (2005). Energy Efficient Information Collection with the ARIMA model in Wireless Sensor Networks. IEEE Communications.

Moghadam, R. A. and Keshmirpour, M. (2011). Hybrid ARIMA and Neural Network Model for Measurement stimation in Energy-Efficient Wireless Sensor Networks. In Informatics Engineering and Information Science, PT III, volume 253 of Communications in Computer and Information Science, pages 35-48.

Pai, P. and Hong, W. (2005). Support vector machines with simulated annealing algorithms in electricity load forecasting. Energy Conversion and Management, 46(17):2669-2688.

Pai, P. and Lin, C. (2005). A hybrid ARIMA and support vector machines model in stock price forecasting. Omega-International Journal of Management Science, 33(6):497505 .

Ruela, A. S., Cabral, R. S., and Aquino, Andre L. L. Guimaraes, F. G. (2009). Evolutionary design of wireless sensor networks based on complex networks. In Proceedings of the 2009 Fifth International Conference on Intelligent Sensors, Sensor Networks and Information Processing, pages 237-242.

Thamizhselvi, S. and Mary, P. S. (2016). A Survey about Data Prediction in Wireless Sensor Networks with Improved Energy Efficiency. Research Journal of OF Pharmaceutical Biological and Chemical Sciences, 7(2):2118-2120.

Wang, X., Wang, S., Ma, J., and Bi, D. (2008). Energy efficient organization of wireless sensor networks with adaptive forecasting. SENSORS, 8(4):2604-2616.

Xiao-Ying, D., Ding-Hui, Y., Tao, L., and Jing, X. (2009). Study on Mercer condition extension of support vector regression based on Ricker wavelet kernel. Chinese Journal of Geophysics-Chinese Edition, 52(9):2335-2344.

Yuan, C., Liu, S., and Fang, Z. (2016). Comparison of china's primary energy consumption forecasting by using arima (the autoregressive integrated moving average) model and $\operatorname{gm}(1,1)$ model. Energy, 100:384 - 390 .

Zhang, G. (2003). Time series forecasting using a hybrid ARIMA and neural network model. Neurocomputing, 50(Supplement C):159 - 175.

Zhang, G., Patuwo, E., and Hu, M. Y. (1998). Forecasting with artificial neural networks: the state of the art. International Journal of Forecasting, 14(14):35-62. 